\title{
Physiological characterization of a novel PPAR pan agonist, 2-(4-(5,6-methylenedioxybenzo[d]thiazol-2-yl)-2- methylphenoxy)-2-methylpropanoic acid (MHY2013)
}

\author{
Hye Jin An ${ }^{1,2}$, Bonggi Lee ${ }^{1,2,5}$, Dae Hyun Kim ${ }^{1,2}$, Eun Kyeong Lee ${ }^{1,2}$, Ki Wung Chung ${ }^{1,2}$, \\ Min Hi Park ${ }^{1,2}$, Hyoung Oh Jeong ${ }^{1,2}$, Sung Min Kim ${ }^{1,2}$, Kyoung Mi Moon ${ }^{1,2}$, Ye Ra Kim ${ }^{1,2}$, \\ Seong Jin Kim ${ }^{1,2}$, Hwi Young Yunn ${ }^{1,2}$, Pusoon Chun ${ }^{3}$, Byung Pal $\mathrm{Yu}^{4}$, Hyung Ryong \\ Moon $^{1,2}$, Hae Young Chung ${ }^{1,2}$ \\ ${ }^{1}$ College of Pharmacy, Pusan National University, Busan 46241, Republic of Korea \\ ${ }^{2}$ Molecular Inflammation Research Center for Aging Intervention (MRCA), Pusan National University, Busan 46241, Republic of Korea \\ ${ }^{3}$ College of Pharmacy, Inje University, Gyeongsangnam-do 50834, Republic of Korea \\ ${ }^{4}$ Department of Physiology, The University of Texas Health Science Center at San Antonio, San Antonio, TX 78229-3900, USA \\ ${ }^{5}$ Korean Medicine (KM)-Application Center, Korea Institute of Oriental Medicine (KIOM), Daegu 41062, Republic of Korea \\ Correspondence to: Hae Young Chung, email: hyjung@pusan.ac.kr \\ Hyung Ryong Moon, email: mhr108@pusan.ac.kr \\ Keywords: MHY2013, PPAR pan agonist, metabolic syndrome, FGF21, adiponectin
}

Received: July 29, $2016 \quad$ Accepted: December 27, $2016 \quad$ Published: January 25, 2017

\section{ABSTRACT}

Recently, agonists targeting multiple peroxisome proliferator-activated receptors (PPARs) have been developed to improve metabolic disorders and minimize the side effects of selective PPAR agonists such as weight gain and dyslipidemia. We newly synthesized six 2-methyl-2-(o-tolyloxy)propanoic acid derivatives based on the structure of a well-known PPAR pan agonist, bezafibrate. Of six compounds, MHY2013 was screened as the strongest activator of three PPAR subtypes based on protein docking simulation and luciferase assays. When treated orally in db/db mice, MHY2013 ameliorated obesity-induced insulin resistance, dyslipidemia, and hepatic steatosis without changes of the body weight and levels of liver and kidney injury markers. MHY2013 decreased the serum triglyceride and fatty acid levels, which is associated with an increase in fatty acid oxidation signaling in the liver and thermogenic signaling on white adipose tissue, respectively. Furthermore, MHY2013 markedly increased serum levels of insulin-sensitizing hormones including fibroblast growth factor 21 (FGF21) and adiponectin. In conclusion, this study suggests that, MHY2013 is a novel PPAR pan agonist that improves obesity-induced insulin resistance, dyslipidemia and hepatic steatosis and elevates insulin-sensitizing hormones in the blood.

\section{INTRODUCTION}

Each PPAR subtype plays a pivotal role in regulating tissue metabolism in hormone-dependent and independent manners [1]. PPAR $\alpha$ is mainly expressed in the liver, muscle, and heart, stimulating $\beta$-oxidation and energy expenditure [2-4], whereas PPAR $\gamma$ is highly expressed in adipose tissue where it regulates adipocyte differentiation and insulin sensitivity [2, 3]. Although biological functions of the ubiquitously expressed PPAR $\beta / \delta$ need to be elucidated, diverse studies have suggested its role in regulating lipid homeostasis and energy utilization. The
PPARs are among the most remarkable targets for the treatment of metabolic syndrome [5]. Consequently, PPAR subtype agonists are in clinical use for metabolic diseases, including type 2 diabetes, cardiovascular diseases, etc. [6]. However, unresolved issues exist for each PPAR subtype agonist. Fibrate-type drugs targeting PPAR $\alpha$ are associated with hepatic toxicity, cholelithiasis, and myopathy, which limit their wider application in patients [7]. In the case of PPAR $\gamma$ selectively agonistic glitazone-type drugs, side effects such as dyslipidemia, heart failure, and weight gain have been reported in clinical stages [7]. Among those, rosiglitazone was even withdrawn in Europe, and 
its use has been restricted in the USA. Clinical and animal studies have demonstrated beneficial effects of PPAR dual and pan agonists, with fewer side effects compared to selective PPAR agonistic drugs, possibly by providing complementary effects [8]. Hence, development of PPAR dual/pan agonistic drugs capable of balanced activation of each PPAR subtype may offer a fascinating therapeutic option. In line with this, we newly synthesized and screened six 2-methyl-2-(o-tolyloxy)propanoic acid derivatives as candidates for PPAR pan agonists and examined their effects on insulin resistance and dyslipidemia in genetically obese $\mathrm{db} / \mathrm{db}$ mice. Our data showed that MHY2013 was the strongest PPAR pan agonist among them. MHY2013 elevated blood FGF21 and adiponectin and increased adipose tissue browning, thereby contributing to improved obesity-induced insulin resistance, hepatic steatosis, and dyslipidemia without affecting body weight. Our study provides a molecular rationale for further development of MHY2013 as treatment of metabolic disorders.

\section{RESULTS}

\section{MHY2013 is a potent PPAR pan agonist in vitro}

To search for powerful PPAR pan agonists, we newly synthesized six 2-methyl-2-(o-tolyloxy)propanoic acid derivatives based on the structure of the well-known PPAR pan agonist bezafibrate (Supplementary Method 1 and Supplementary Table 1 ). The PPRE-luciferase assays for the three PPAR subtypes were performed using YPEN-1 cells to screen for the strongest PPAR pan agonist. Among the six derivatives, MHY2013 had the highest luciferase activities for all PPAR subtypes, and its effect on each PPAR subtype was comparable to that of each specific PPAR subtype agonist (PPAR $\alpha$ : WY14643,
PPAR $\beta / \delta$ : GW501516, and PPAR $\gamma$ : rosiglitazone) (Figure 1). In addition, MHY2013 dose-dependently increased luciferase activities for the three PPAR subtypes, suggesting that the compound strongly activates all PPAR subtypes (Supplementary Figure 1).

\section{MHY2013 can directly bind to three PPAR subtypes}

To investigate whether MHY2013 can bind to the three PPAR subtypes, docking simulation was conducted using the AutoDock 4.2 program. The structure images of the three PPAR subtypes from the docking simulation showed that MHY2013 might directly bind to the three PPAR subtypes (Figure 2A, B, C). To predict the binding energy, the docking topologies of WY14643, GW501516, rosiglitazone, and MHY2013 were simulated to the cocrystal structure of each PPAR subtype. The binding affinities of WY14643, GW501516, and rosiglitazone to $\operatorname{PPAR} \alpha, \operatorname{PPAR} \beta / \delta$, and PPAR $\gamma$, respectively, were -7.93 , -9.68 , and $-8.03 \mathrm{kcal} / \mathrm{mol}$, respectively, whereas the binding affinities of MHY2013 to PPAR $\alpha, \operatorname{PPAR} \beta / \delta$, and PPAR $\gamma$ were $-7.94,-9.2$, and $-8.69 \mathrm{kcal} / \mathrm{mol}$, respectively (Figure 2A, B, C). These results suggest that the binding affinity of MHY2013 to each PPAR subtype may be comparable to that of the positive controls. To predict the binding residues of the three PPAR subtypes, which correlated for each positive control and MHY2013, the LigandScout 3.1 program was used. MHY2013 might form a hydrogen bond with the TYR464A residue and hydrophobic bonds with the ILE354A, PHE273A, LEU460A, LEU456A, and VAL444A residues of PPAR $\alpha$ (Figure 2D). In the case of PPAR $\beta / \delta$, MHY2013 might form hydrogen bonds with the CYS285A and THR292A residues and hydrophobic bonds with the THR268A, ILE333A, LEU330A, and ILE326
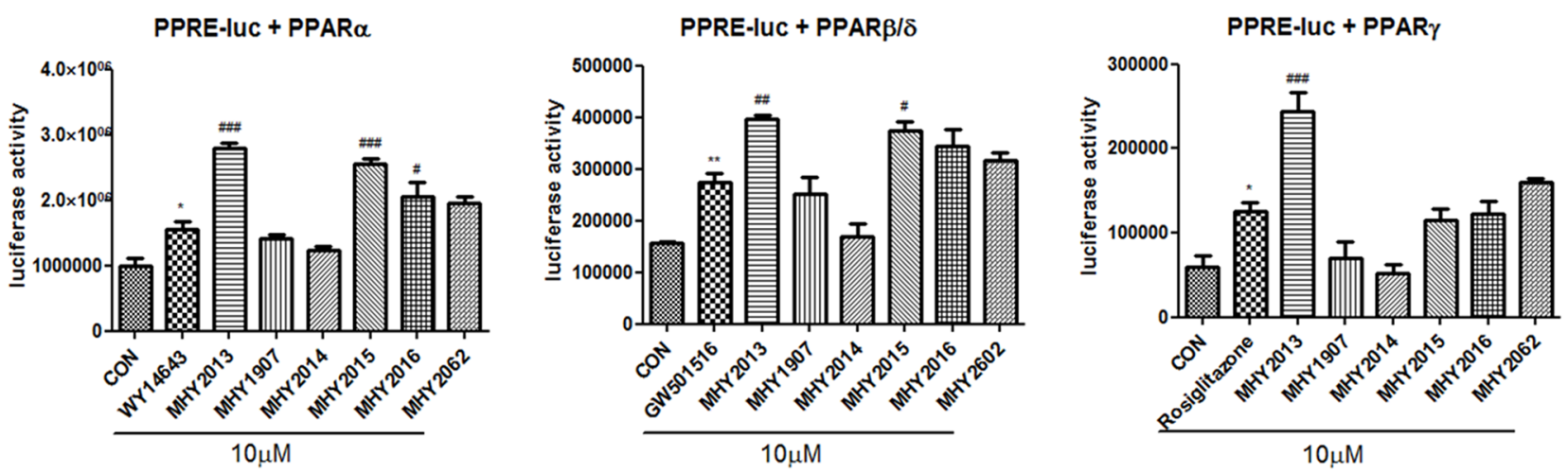

Figure 1: MHY2013 is the strongest PPAR pan agonist among six newly synthesized compounds. For luciferase assays to screen of six newly synthesized compounds for PPAR pan agonist, the 3X-PPRE-TK-LUC plasmid and respective PPAR subtype expression vectors were transfected in YPEN-1 cells. Twenty-four hours after the transfection, the cells were treated with the indicated new compounds or respective PPAR agonists (WY14643, GW501516 or rosiglitazone) for $5 \mathrm{~h}$. The data are shown as the mean \pm SEM ( $=4$ ) luciferase activity, considered to represent the PPAR transcriptional activity. ${ }^{*}, p<0.05$ vs. DMSO control $(\mathrm{CON}) ;{ }^{* *}, p<0.01 \mathrm{vs.} \mathrm{CON}$; ***, $p<0.001$ vs. CON; \#, $p<0.05$ vs. WY14643, GW501516 or rosiglitazone; \#\#, $p<0.01$ vs. WY14643, GW501516 or rosiglitazone; $\# \#$, $p<0.001$ vs. WY14643, GW501516 or rosiglitazone. 
(A)

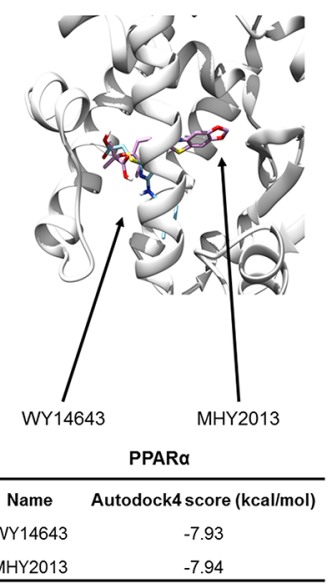

(B)

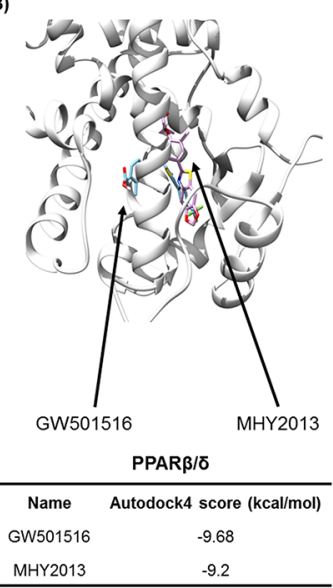

(C)

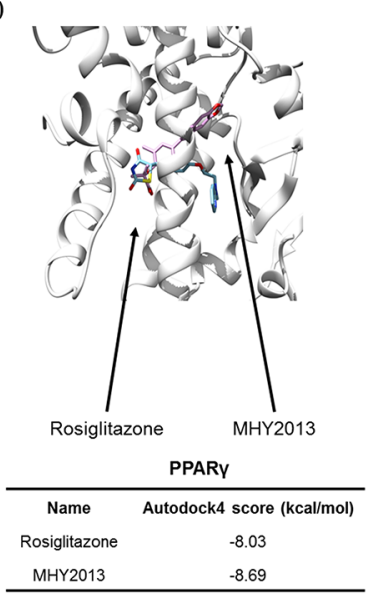

(D)

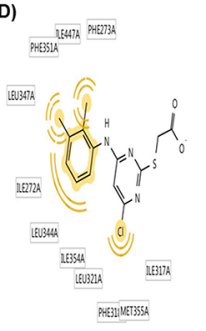

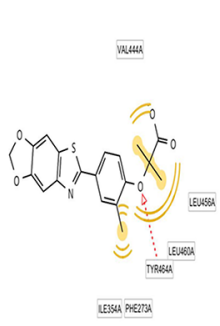

MHY2013
(E)

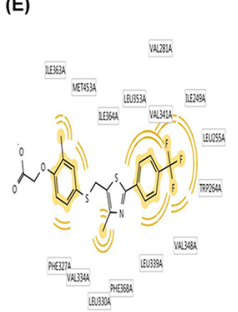

(F)
(G)
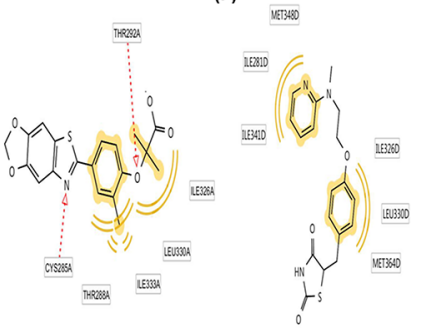

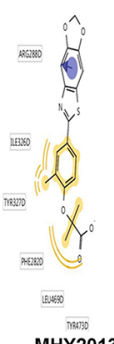

WY14643 PPARa

GW501516
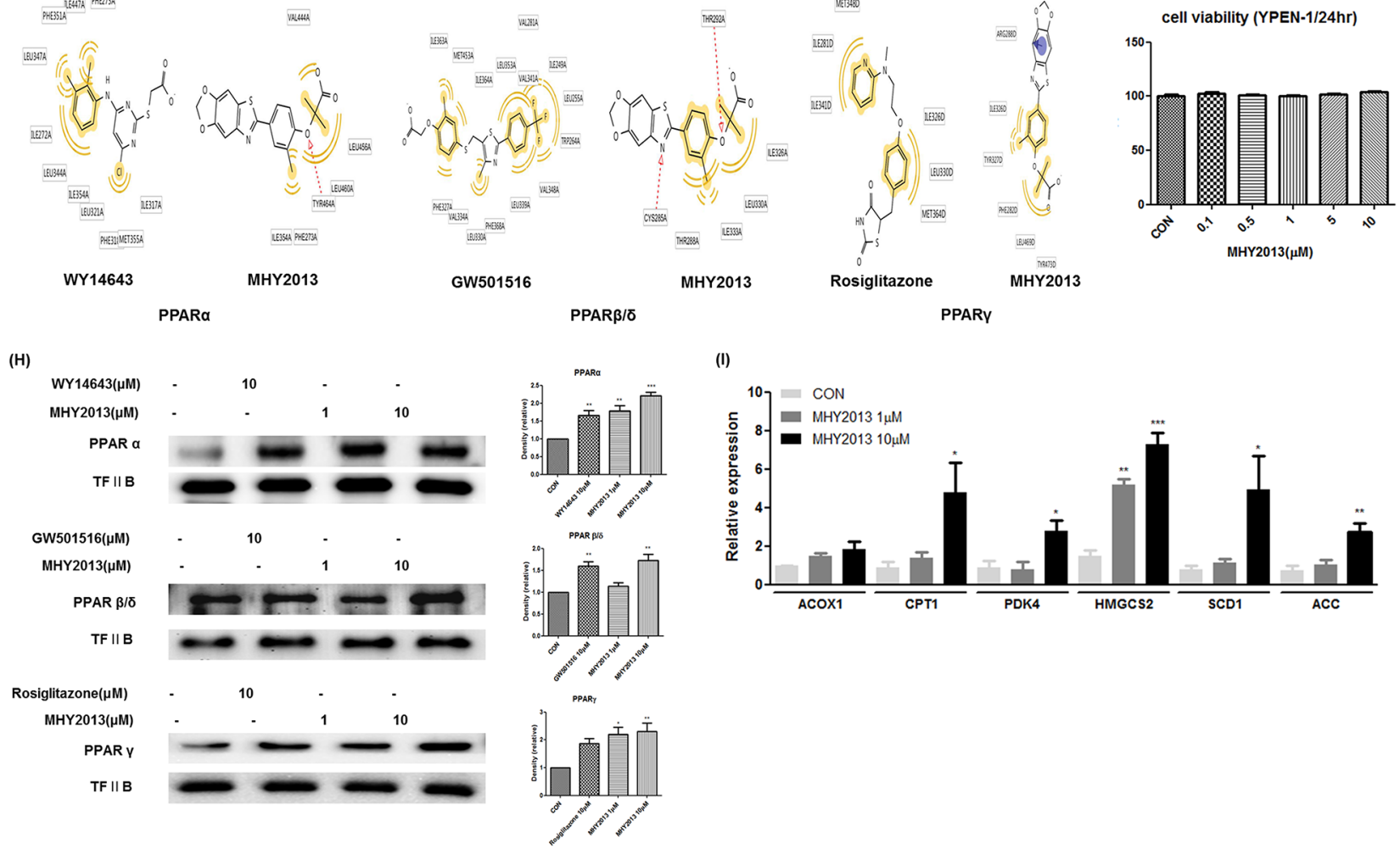

(I)

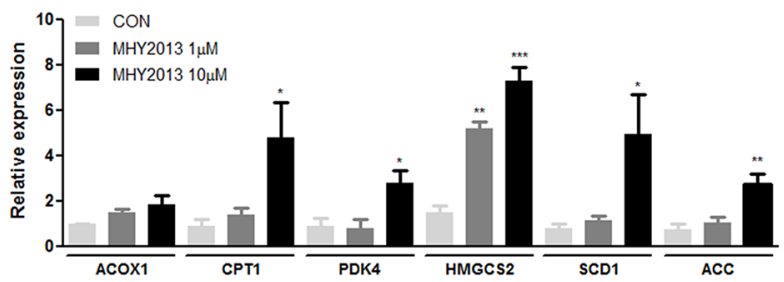

Figure 2: MHY2013 can directly bind to the three PPAR subtypes and increases their transcriptional activity. A, B, C. Computational structure prediction for docking simulation between each of the three PPAR subtypes and MHY2013. The gray zone indicates the active site of PPAR subtypes (PDB ID: PPAR $\alpha, 1 \mathrm{~K} 7 \mathrm{~L}$; PPAR $\beta / \delta, 1 \mathrm{GWX}$; and PPAR $\gamma, 3 \mathrm{DZY}$ ), magenta indicates MHY2013 and cyan indicates the respective positive control for each of the three PPAR subtypes. The small tables indicate the predicted docking score of MHY2013 and respective positive control with three PPAR subtypes. D, E, F. The binding sites of MHY2013 and the respective positive control for each of the three PPAR subtype are shown. The yellow indicates the hydrophobic interaction, purple indicates the aromatic interaction, and red indicates the hydrogen bond. G. YPEN-1 cells were treated with various concentrations $(0.1,0.5,1,5$ and $10 \mu \mathrm{M})$ of MHY2013 for $24 \mathrm{~h}$, and then cell viability assay was performed $(\mathrm{n}=4)$. H. The nuclear levels of PPARs in MHY2013-treated cells for 90 min were analyzed by western blotting, and the blots were quantified by densitometry $(\mathrm{n}=3)$. Transcription factor II B (TF II B) was used as the loading control. I. Levels of PPAR target genes (PPARa: $A C O X 1$ and $C P T 1$, PPAR $\beta / \delta: P D K 4$ and HMGCS2, PPAR $\gamma: S C D 1$ and $A C C$ ) in MHY2013-treated YPEN-1 cells for $5 \mathrm{~h}$ were analyzed by quantitative reverse transcription-polymerase chain reaction (qRT-PCR). The data are shown as the mean $\pm \operatorname{SEM}(\mathrm{n}=3) .{ }^{*}, p<0.05$ vs. $\mathrm{CON} ;{ }^{* *}, p<0.01$ vs. $\mathrm{CON} ;{ }^{* *}, p<0.001$ vs. CON. 
residues (Figure 2E). MHY2013 might interact with PPAR $\gamma$ by forming an aromatic bond with the ARG288D residue and hydrophobic bonds with the ILE326D, TYR327D, PHE282D, LEU469D, and TYR473D residues (Figure 2F). In particular, MHY2013 formed hydrophobic bonds with the ILE354A residue of PPAR $\alpha$, LEU330A residue of PPAR $\beta / \delta$, and ILE326D residue of PPAR $\gamma$, in common with each PPAR subtype agonist (Figure 2D, E, F). These data indicate that MHY2013 may directly bind to all PPAR subtypes with affinities comparable to those of well-known PPAR subtype agonists.

\section{MHY2013 increases transcriptional activity of three PPAR subtypes}

To investigate whether MHY2013 has cytotoxicity that was reported in some well-known PPAR agonists, including fenofibrate and pioglitazone, we carried out 3-(4,5-dimethylthiazol-2-yl)-2,5-diphenyltetrazolium bromide (MTT) assays and proved that MHY2013 had no cytotoxicity (up to $10 \mu \mathrm{M}$ ) in YEPN-1 cells during $24 \mathrm{~h}$ incubation (Figure 2G). Because PPARs activated by ligand binding are translocated to the nucleus, we investigated whether MHY2013 induced translocation of PPARs to the nucleus. Immunofluorescence staining revealed that the nuclear distribution of the three PPAR subtypes were increased in the MHY2013-treated groups compared with those treated with the vehicle (DMSO) and each PPAR positive control (Supplementary Figure 2). Furthermore, western blotting results showed that the nuclear protein levels of the three PPAR subtypes were increased dose-dependently by the MHY2013 treatment (Figure 2H). Consistently, the mRNA levels of the target genes of PPAR $\alpha(A C O X 1$ and $C P T 1), \mathrm{PPAR} \beta / \delta$ (PDK4 and HMGCS2), and PPAR $\gamma$ (SCD1 and $A C C$ ) were significantly elevated by the MHY2013 treatment (Figure 2I). These results confirm that MHY2013 is a strong PPAR pan agonist targeting all PPAR subtypes.

\section{Effects of MHY2013 on blood profile and insulin sensitivity in obesity model}

Obesity is generally accompanied by severe alterations in blood nutrient and metabolite profiles, which are closely related to metabolic syndrome, including insulin resistance and dyslipidemia. To evaluate the beneficial effects of MHY2013 on the blood profile, genetically obese $\mathrm{db} / \mathrm{db}$ mice were administered with MHY2013 (5 mg/kg/day) by oral gavage for three weeks. There were no differences in the food intake and body weight between the groups (Figure 3A, B). We first tested whether MHY2013 had toxic effects in vivo. Serum aspartate transaminase (AST) and alanine transaminase (ALT) level used to detect the liver injury and the serum creatinine level used to detect the renal injury were unaffected by the MHY2013 treatment (Figure 3C, D, E), suggesting that MHY2013 has no severe toxicity for the liver and kidney. Then, we examined its effect on the blood profile associated with glucose and lipid metabolism. As expected, triglyceride (TG), non-esterified fatty acid (NEFA), fasting glucose, and circulating insulin levels highly increased in the $\mathrm{db} / \mathrm{db}$ mice compared with the $\mathrm{db} / \mathrm{m}$ mice, confirming the development of dyslipidemia and insulin resistance in the $\mathrm{db} / \mathrm{db}$ mice (Figure $3 \mathrm{~F}, \mathrm{G}$, I, J). However, MHY2013 reduced the serum levels of TGs and NEFAs in the $\mathrm{db} / \mathrm{db}$ mice (Figure 3F, G). The GTT showed that MHY2013 improved the obesityinduced glucose intolerance (Figure 3H). Moreover, the MHY2013 treatment significantly reduced the fasting blood glucose and serum insulin levels in the $\mathrm{db} / \mathrm{db}$ mice, without affecting the body weight (Figure 3I, J). These results suggest that MHY2013 improves obesity-induced dyslipidemia and insulin resistance, and the improvement is not a secondary effect of the body weight reduction.

\section{Effects of MHY2013 on liver of obese mice}

Hepatic steatosis is commonly found together with insulin resistance in obesity. We examined the beneficial effect of MHY2013 on the liver and found that the compound ameliorated the obesity-induced hepatic steatosis as evidenced by a significant decrease in the hepatic TG concentration (Figure 4A). As a potential factor underlying the MHY2013-mediated decrease in the hepatic TG level, we investigated AMP-activated protein kinase $(\mathrm{AMPK}) / \beta$-oxidation signaling because diverse studies have indicated that all PPAR subtypes induce AMPK activation through various mechanisms, such as a physical interaction, phosphorylation, and upregulation of AMPK activation-related proteins [9]. Phosphorylation of AMPKa1/2 (Thr172) was significantly increased in the liver but not adipose tissue and muscle of the MHY2013treated $\mathrm{db} / \mathrm{db}$ mice compared to those of the vehicle-treated $\mathrm{db} / \mathrm{db}$ mice (Figure 4B and Supplementary Figure 3A, B). Moreover, MHY2013 induced phosphorylation of LKB1 (Ser431) known as upstream of AMPK in the liver (Figure 4C). Consistently, the mRNA expression levels of fatty acid oxidation-related genes, including $A C O X 1, C P T 1$, $H M G C S 2$, and $P D K 4$, were increased in the livers of the MHY2013-treated db/db mice (Figure 4D), indicating that the increased AMPK/ $\beta$-oxidation signaling contributes to the improvement of hepatic steatosis.

FGF21 is a recently described hepatokine, primarily regulated by $\operatorname{PPAR} \alpha$. Numerous studies have revealed the pharmaceutical importance of FGF21 for treating metabolic syndrome, which is due to its strong insulin-sensitizing and thermogenic effects on adipose tissues [10]. Because it is possible that MHY2013 activates PPAR $\alpha$ via direct binding, we examined whether the compound stimulated the FGF21 production in the liver. The data showed that the mRNA expression 
(A)

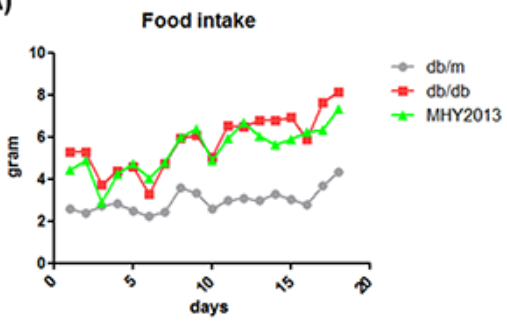

(B)

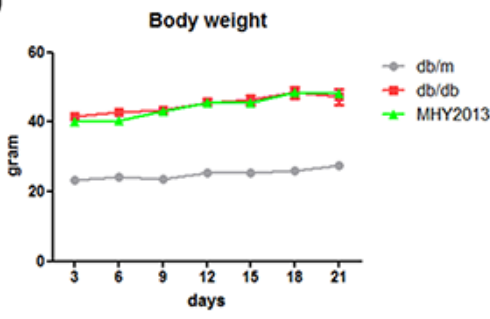

(C)

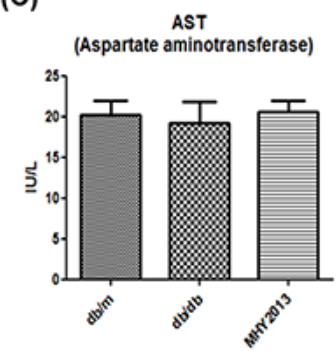

(F)

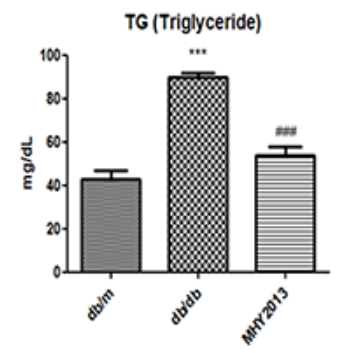

(D)

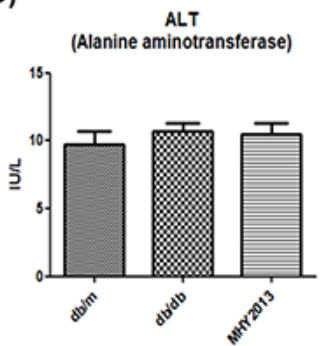

(G)

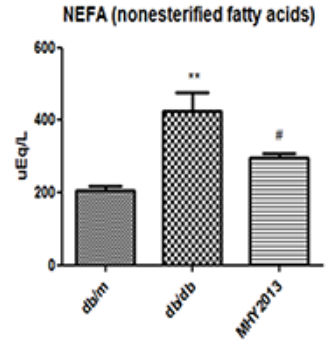

(E)

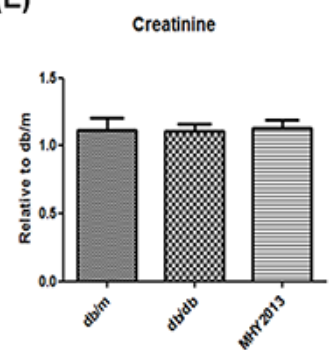

(H)
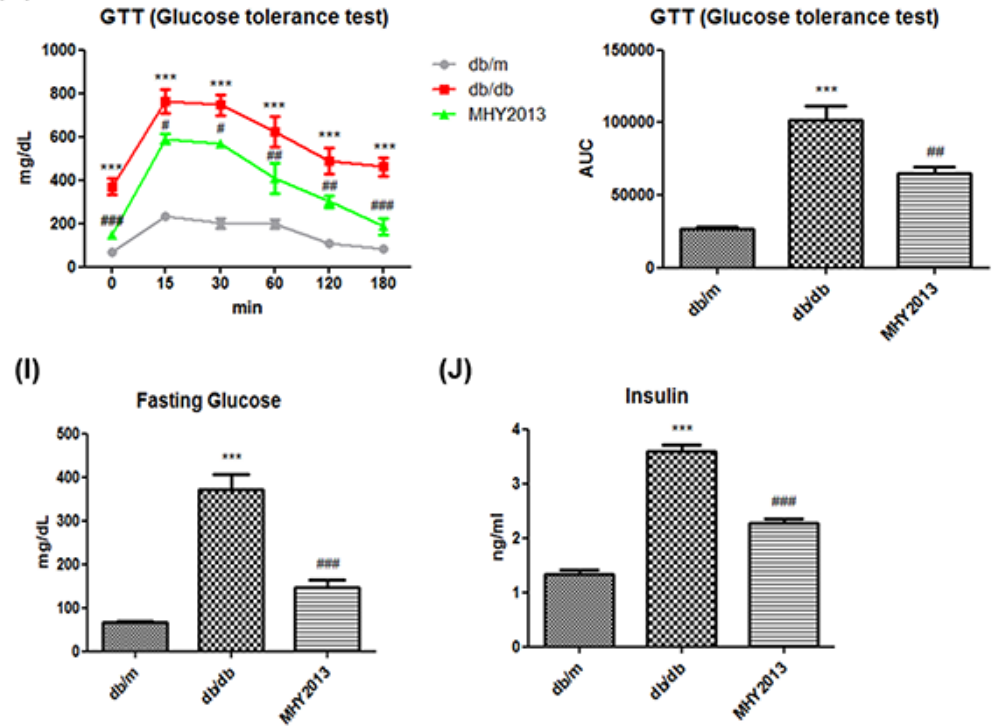

(J)

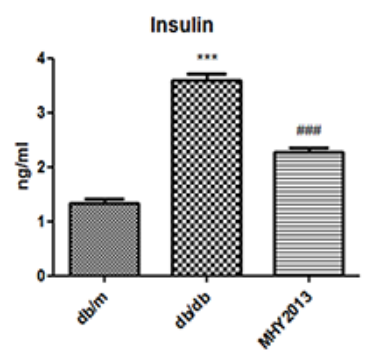

Figure 3: MHY2013 exhibits potent glucose- and lipid-lowering effects without changing the body weight in genetically obese $\mathbf{~ d b} / \mathbf{d b}$ mice. The mice were orally treated with the vehicle (water) or $5 \mathrm{mg} / \mathrm{kg} /$ day of MHY2013 for three weeks $(\mathrm{n}=5)$. A. Food intake. B. Body weight. C. Serum aspartate aminotransferase (AST), D. alanine aminotransferase (ALT), E. creatinine, F. triglyceride (TG), and G. non-esterified fatty acid (NEFA) levels. H. Glucose concentrations were determined using an intraperitoneal glucose tolerance test (ipGTT) [inset: area under the curve (AUC), $\mathrm{mg} / \mathrm{dL} \times \mathrm{min}$ ] by administration of $1.5 \mathrm{~g} / \mathrm{kg}$ glucose after overnight fasting. I. Fasting serum glucose and $\mathbf{J}$. insulin levels. The data are shown as the mean $\pm \operatorname{SEM}(\mathrm{n}=5) .{ }^{*}, p<0.05 \mathrm{vs} . \mathrm{db} / \mathrm{m} ;{ }^{* *}, p<0.01 \mathrm{vs} . \mathrm{db} / \mathrm{m} ;{ }^{* * *}, p<0.001$ vs. db/m; $\#, p<0.05$ vs. db/db; \#, $p<0.01$ vs. db/db; \#\#, $p<0.001$ vs. db/db. 
levels of FGF21 were markedly increased in the livers of the $\mathrm{db} / \mathrm{db}$ mice by the MHY2013 treatment (Figure 4E). Consistently, the serum level of FGF21 was also elevated by MHY2013 (Figure 4F). The FGF21luciferase assay using HepG2 cells further revealed that MHY2013 highly increased the FGF21-luciferase activity to the level comparable to that of the PPAR $\alpha$ agonist WY14643-treated group (Figure 4G). These data indicate that the MHY2013-mediated activation of
$\operatorname{PPAR} \alpha$ transcriptionally upregulates FGF21 in the liver, contributing to the FGF21 increase in blood.

\section{Effects of MHY2013 on adipose tissue of obese mice}

Although their expression levels are different, all PPAR subtypes have been shown to affect adipocyte metabolism. Indeed, a strong thermogenic effect of FGF21
(A)

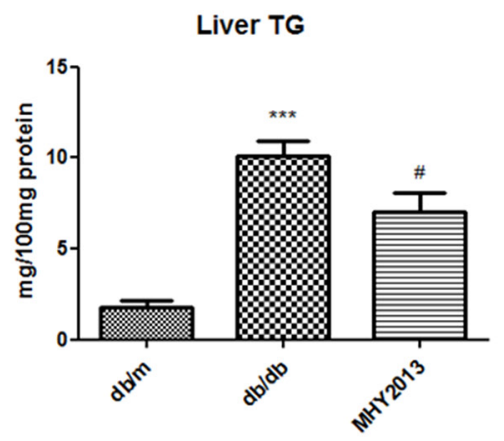

(C)

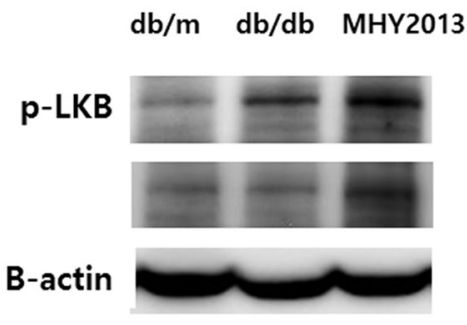

(E)

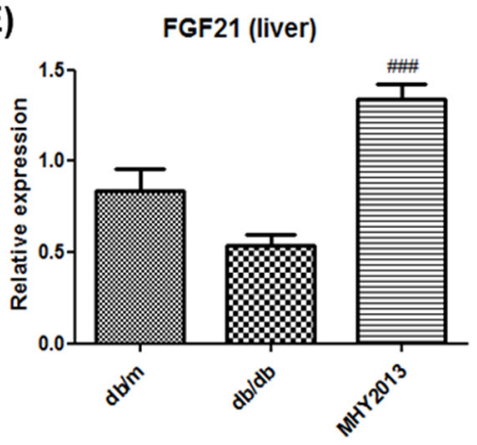

(B)

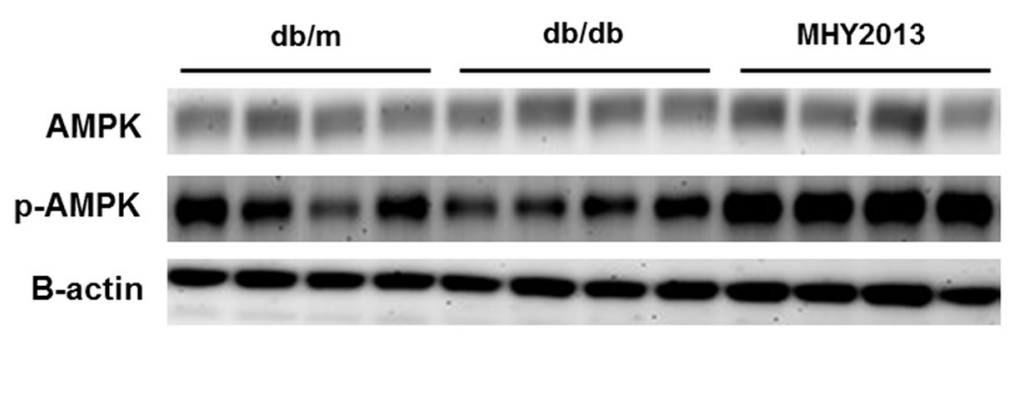

(D)

Mitochondria FA $\beta$-oxidation

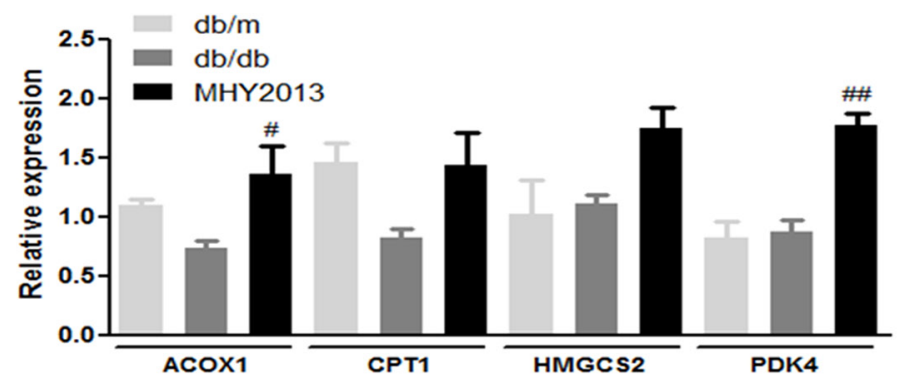

(F)

FGF21 (serum)

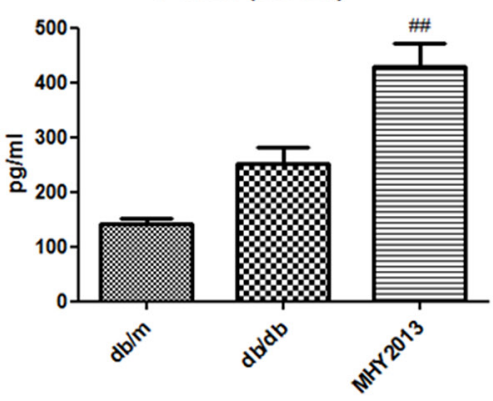

(G)

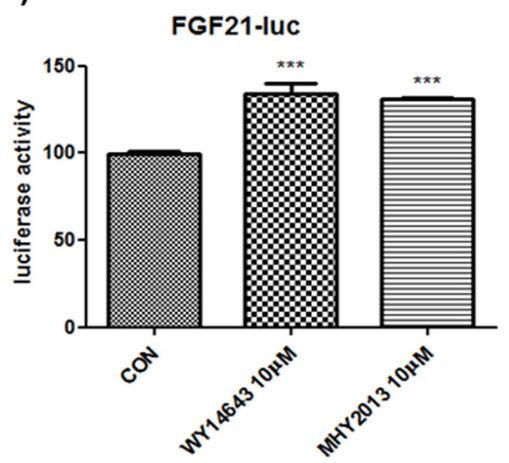

Figure 4: MHY2013 ameliorates hepatic steatosis and increases serum FGF21 levels. The mice were orally treated with vehicle (water) or $5 \mathrm{mg} / \mathrm{kg} /$ day of MHY2013 for three weeks $(\mathrm{n}=5)$. A. Hepatic triglyceride (TG) contents. B. Activated p-AMPK (Thr172), total AMPK and C. p-LKB1 (Ser431) were analyzed in liver tissue by western blotting. $\beta$-Actin was used as the loading control. D. mRNA levels of fatty acid oxidation-related genes ( $A C O X 1, C P T 1, H M G C S 2$, and PDK4) were analyzed in liver tissue by qRT-PCR. E. mRNA levels of fibroblast growth factor 21 (FGF21) in liver tissue were analyzed by qRT-PCR. F. Serum FGF21 levels were examined using the Mouse/Rat FGF-21 Quantikine ELISA Kit. The data are shown as the mean \pm SEM $(\mathrm{n}=5)$.,$p<0.05 \mathrm{vs} . \mathrm{db} / \mathrm{m} ; * *, p<0.01 \mathrm{vs.} \mathrm{db} / \mathrm{m}$; ***, $p<0.001 \mathrm{vs}$. $\mathrm{db} / \mathrm{m}$; \#, $p<0.05$ vs. db/db; \#\#, $p<0.01 \mathrm{vs.} \mathrm{db/db;} \mathrm{\# \# ,} p<0.001$ vs. db/db. G. The FGF21-LUC plasmid was transfected into HepG2 cells. Twenty-four hours after the transfection, cells were treated with the indicated compound for $5 \mathrm{~h}$. The data are shown as the mean $\pm \mathrm{SEM}(\mathrm{n}=5)$ luciferase activity, considered to represent the PPAR transcriptional activity. ${ }^{*}, p<0.05 \mathrm{vs}$. CON; ${ }^{* *}, p<0.01 \mathrm{vs.} \mathrm{CON}$; ${ }^{* * *}, p<0.001 \mathrm{vs.} \mathrm{CON}$. 

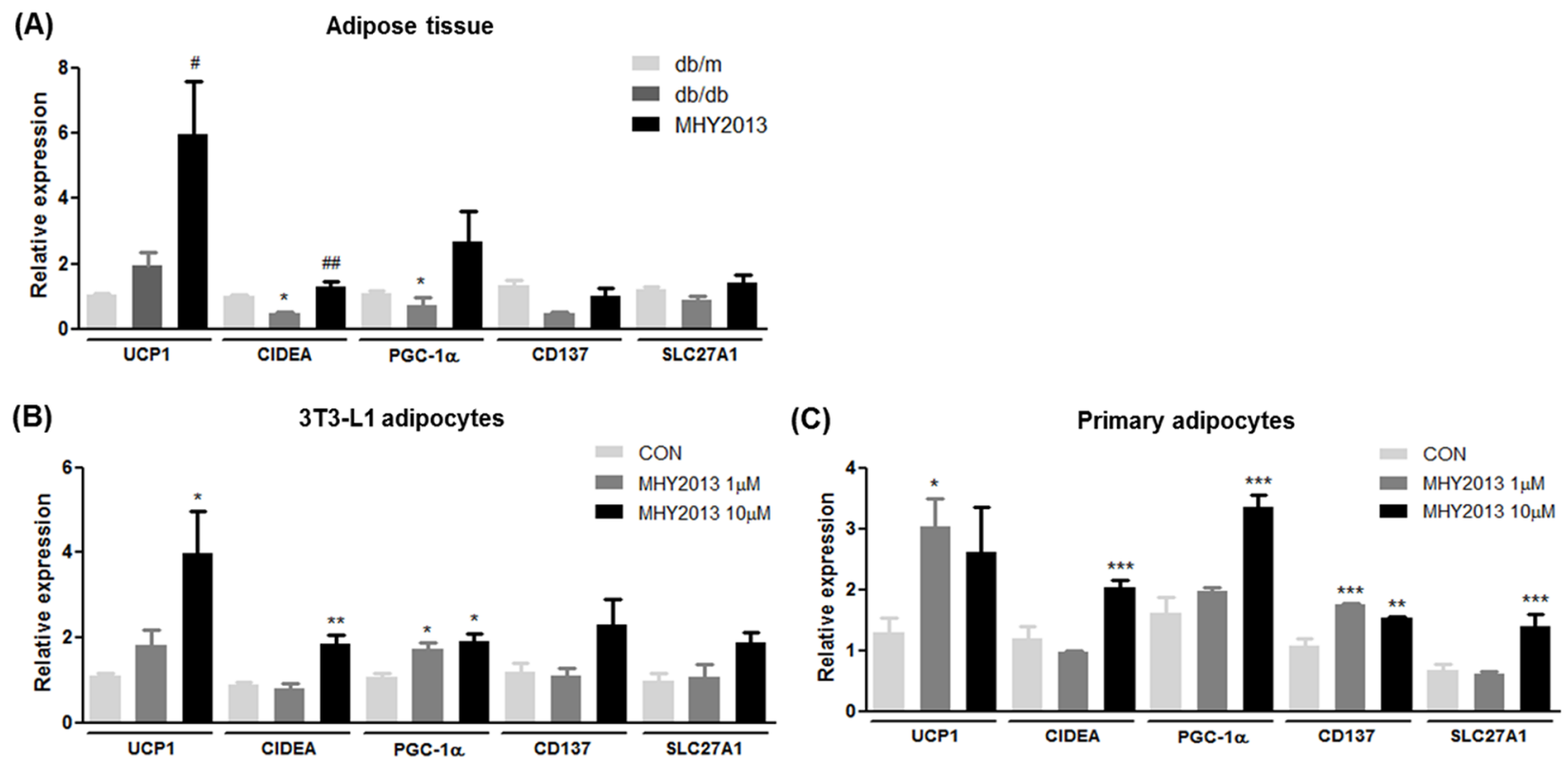

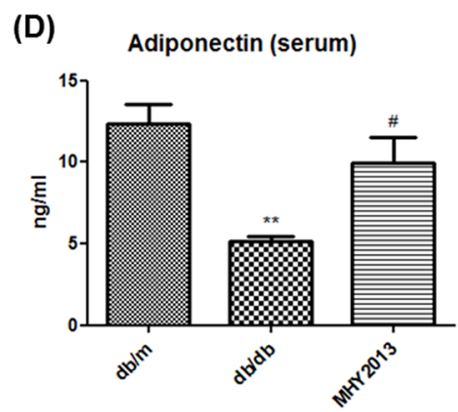

(G)

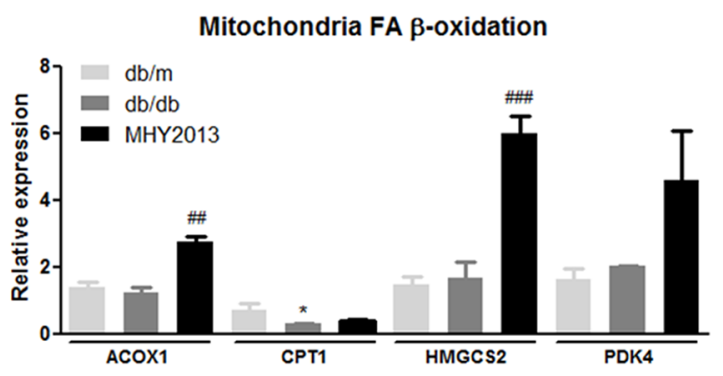

(E)

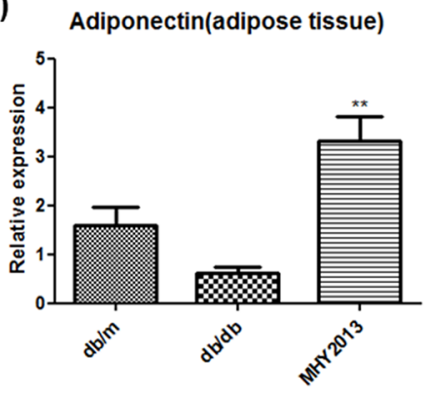

(F)

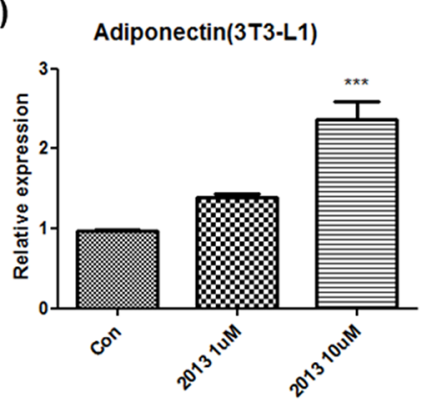

(H)

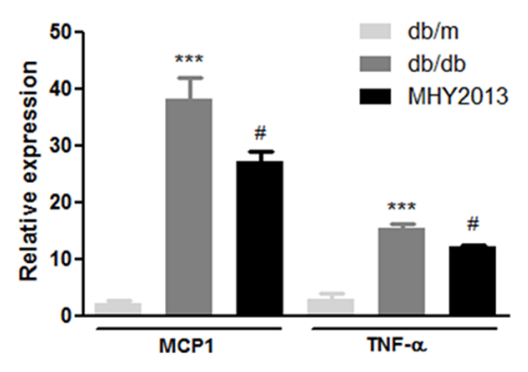

Figure 5: MHY2013 promotes adipose tissue browning and increases adiponectin levels. The mice were orally treated with the vehicle (water) or $5 \mathrm{mg} / \mathrm{kg} /$ day of MHY2013 for three weeks $(\mathrm{n}=5$ ). A. In adipose tissue B. 3T3-L1 adipocytes and C. primary adipocyte from subcutaneous fat, mRNA expression of browning markers (UCP1, CIDEA, PGC-1 $\alpha, C D 137$, and SLC27A1) was examined by qRT-PCR. D. In adipose tissue, mRNA expression of adiponectin was evaluated by qRT-PCR. E. Serum adiponectin levels were analyzed using the Mouse Adiponectin ELISA Kit. F. In 3T3-L1 cells, mRNA expression of adiponectin was evaluated by qRT-PCR. In adipose tissue, the mRNA levels of $\mathbf{G}$. fatty acid oxidation-related genes (ACOX1, CPT1, HMGCS2, and PDK4) and $\mathbf{H}$. inflammatory cytokines $(M C P 1$ and $T N F-\alpha)$ were analyzed by qRT-PCR. For mouse studies, ${ }^{*}, p<0.05 \mathrm{vs.} \mathrm{db/m;} * *, p<0.01 \mathrm{vs.} \mathrm{db/m;} \mathrm{***,} p<0.001$ vs. db/m; \#, $p<0.05$ vs. db/db; \#\#, $p<0.01$ vs. db/db; \#\#, $p<0.001$ vs. db/db. For cell experiments, ${ }^{*}, p<0.05$ vs. CON; ${ }^{* *}, p<0.01$ vs. $\mathrm{CON} ;{ }^{* * *}, p<0.001$ vs. $\mathrm{CON}$. 
on adipose tissues has been well established. To reveal the net effects of MHY2013 on white adipose tissue (WAT), we examined various signaling pathways related to fatty acid oxidation, thermogenesis, and inflammation.

The mRNA expression levels of browning markers such as UCP1 and CIDEA were significantly increased in adipose tissue of the MHY2013-treated db/db mice compared with the vehicle-treated $\mathrm{db} / \mathrm{db}$ mice (Figure 5A). Other browning markers, such as PGC1- $\alpha, C D 137$, and SLC27A1, also showed a tendency to be slightly increased by MHY2013 (Figure 5A). To check whether MHY2013 directly induces the browning of white adipocytes, 3T3-L1 pre-adipocytes and primary adipocytes isolated from subcutaneous fat of C57/BL6 mice were differentiated into adipocytes for seven days with or without MHY2013. As a result, MHY2013 dose-dependently increased the mRNA levels of browning markers such as UCP1, CIDEA, and PGC1- $\alpha$ in the 3T3-L1 and primary adipocytes (Figure $5 \mathrm{~B}, \mathrm{C}$ ). To further examine the adipose browning effect of MHY2013, we observed the morphology of lipid droplets because brown adipocytes are characterized by a multi-locular lipid droplet structure. MHY2013 appeared to alter the morphology of adipocytes toward a multi-locular lipid droplet structure in 3T3-L1 cells (Supplementary Figure 4A). These data suggest that MHY2013 induces browning of WAT.

One of the important functions of adipose tissue is secretion of adipokines to regulate systemic nutrient metabolism. Adiponectin is probably one of the bestknown adipokines, which elevates the insulin sensitivity and fatty acid oxidation and reduces the inflammation. Indeed, it has been shown that beneficial effects of PPAR subtype agonists are partially mediated via increasing adiponectin levels. Because adiponectin gene expression in adipocytes is induced by all three PPAR subtypes, we evaluated adiponectin levels in the MHY2013-treated $\mathrm{db} / \mathrm{db}$ mice. MHY2013 notably increased the mRNA expression level of adiponectin in adipose tissue (Figure 5D). Consistently, the blood adiponectin level was higher in the MHY2013-treated than in the vehicle-treated db/ $\mathrm{db}$ mice (Figure 5E). To investigate whether MHY2013 directly regulates the level of adiponectin in adipocytes, we measured the adiponectin mRNA expression level in MHY2013-treated 3T3-L1 adipocytes. The mRNA expression of adiponectin was greatly increased by MHY2013 in the 3T3-L1 cells (Figure 5F). These data demonstrate that the MHY2013-mediated increase in adiponectin levels contributes to the improvement of obesity-related insulin resistance and dyslipidemia.

To further examine the effects of MHY2013 on adipose tissue, we determined the mRNA expression levels of genes associated with fatty acid oxidation and inflammation signaling pathways. The mRNA expression levels of fatty acid oxidation-related genes (ACOX1,CPT1, $H M G C S 2$, and $P D K 4$ ) were increased in adipose tissue of the MHY2013-treated $\mathrm{db} / \mathrm{db}$ mice and 3T3-L1 cells (Figure 5G and Supplementary Figure 4B). MHY2013 also reduced the mRNA expression levels of inflammatory genes such as MCP1 and TNF- $\alpha$ (Figure 5H). Although we showed that the MHY2013 treatment increased the FGF21 expression in the liver, no significant change in FGF21 expression was observed in adipose tissue (Supplementary Figure 4C). Together, these data indicate that MHY2013 not only stimulates the metabolic pathways for energy expenditure but also inhibits inflammatory signaling, thereby contributing to maintaining metabolically healthy adipose tissue.

\section{Effects of MHY2013 on skeletal muscle of obese mice}

To investigate whether MHY2013 has beneficial effects on skeletal muscle, we measured mRNA
(A)

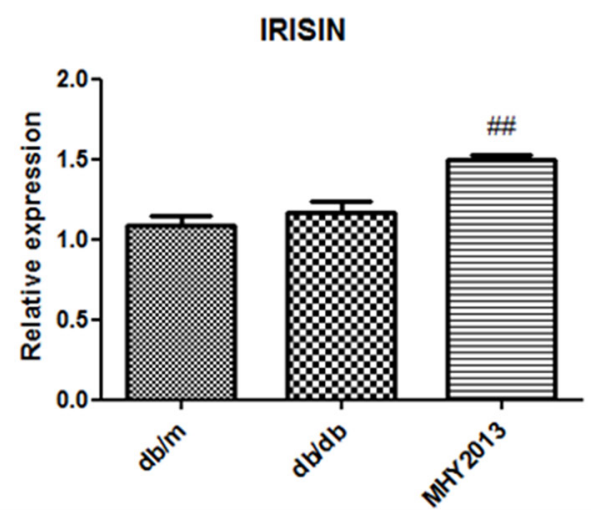

(B)

\section{Mitochondria FA $\beta$-oxidation}

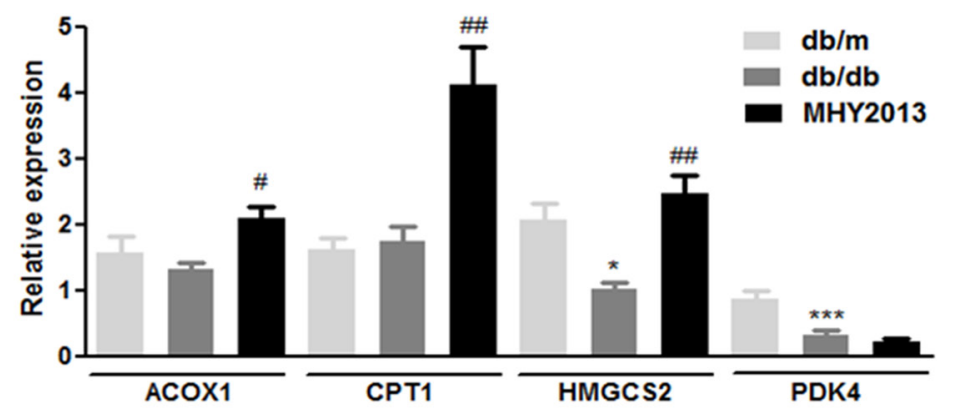

Figure 6: MHY2013 increased mRNA expression of irisin and fatty acid oxidation-related genes. The mice were orally treated with the vehicle (water) or $5 \mathrm{mg} / \mathrm{kg} /$ day of MHY2013 for three weeks $(\mathrm{n}=5)$. In muscle, mRNA levels of A. IRSIN and B. fatty acid oxidation-related genes ( $A C O X 1, C P T 1, H M G C S 2$, and PDK4) were analyzed by qRT-PCR. *, $p<0.05 \mathrm{vs.} \mathrm{db/m;} \mathrm{**,} p<0.01 \mathrm{vs.} \mathrm{db/m;}$ ***, $p<0.001$ vs. db/m; \#, $p<0.05$ vs. db/db; \#\#, $p<0.01$ vs. db/db; \#\#, $p<0.001$ vs. db/db. 
expression levels of fatty acid oxidation-related genes and irisin, a recently identified myokine that improves obesityrelated metabolic syndrome. MHY2013 increased mRNA expression levels of ACOX1, CPT1, HMGCS2, and irisin in the skeletal muscle (Figure 6A and 6B). Although further studies are necessary for proving beneficial effects of MHY2013 on skeletal muscle, these data shows that MHY2013 may increase fatty acid oxidation and irisin production in skeletal muscle.

\section{DISCUSSION}

Through in silico docking simulation and multiple biological analysis, we screened MHY2013 as the strongest agonist for all PPAR subtypes among six 2-methyl-2-(o-tolyloxy)propanoic acid derivatives. When injected orally in db/db mice, MHY2013 greatly improved obesity-induced insulin resistance, dyslipidemia, and hepatic steatosis without a change in body weight and apparent signs of hepatic or renal toxicity. Although the underlying mechanisms should be diverse considering numerous effects of PPARs in multiple tissues, the elevation of beneficial hormones including FGF21 and adiponectin, WAT browning signaling, and fatty acid oxidation signaling in liver and skeletal muscle may synergistically contribute to the beneficial effects of MHY2013. Thus, MHY2013 may be a novel pharmaceutical agent that can be used for intervention in obesity-induced metabolic syndrome.

Previous studies have revealed that there are major drawbacks in selective PPAR agonists, such as a body weight gain and dyslipidemia. As an alternative, drugs targeting multiple PPARs have been developed. Although bezafibrate was first clinically tested as a PPAR pan agonist to improve metabolic disorders [11, 12], they caused some mooted points like severe hepatic and renal toxicity and low potency $[13,14]$. Our data showed that MHY2013 did not accompany the known side effects including body weight gain and hepatic and renal toxicity. When compared to a PPAR $\alpha / \gamma$ dual agonist MHY908 $[15,16]$, MHY2013 appears to be a stronger activator for PPARs than MHY908 (unpublished data). We assume that the balanced activation of three PPAR subtypes by MHY2013 may contribute to the beneficial effects on metabolic syndrome with minimizing known side effects by other PPAR agonists.

The PPAR subtypes regulate the expression of endocrine hormones that can influence glucose and lipid metabolism [17]. FGF21 is a hormone secreted from the liver and adipose tissues in response to activation of PPAR $\alpha$ and PPAR $\gamma$ respectively. FGF21 has been considered a promising intervention target for metabolic diseases, including fatty liver, obesity, and diabetes due to its insulin-sensitizing and thermogenesis-inducing effects [18]. Our data showed that FGF21 was increased in the liver and serum of the MHY2013-treated $\mathrm{db} / \mathrm{db}$ mice, but the mRNA expression of FGF21 did not change in the the WAT. Therefore, we assume that the liver is the main contributor to the increase in serum FGF21 level by MHY2013 treatment. Besides FGF21, a well-known insulin-sensitizing hormone adiponectin is also increased in the serum of the MHY2013-treated mice possibly due to the upregulation of adiponectin expression in WAT. Considering MHY2013 elevated the mRNA expression of adiponectin in 3T3-L1 adipocyte cells, the compound may directly upregulate adiponectin expression in adipocytes by activating PPAR $\gamma$ that are well-known regulators of adiponectin transcription [19].

Moreover, MHY2013 induced AMPK activation in the liver. Because AMPK activation has been shown to stimulate the energy expenditure, including fatty acid oxidation, the MHY2013-mediated increase in fatty acid oxidation signaling is likely associated with the AMPK activation in the liver. Furthermore, it is very likely that the utilization of fatty acids in the liver may contribute to the reduced TG level in circulation in the MHY2013-treated mice because it has been reported that the increased fatty acid oxidation in the liver lowers serum lipid levels [20].

Adipose tissue browning is important for ameliorating obesity-related metabolic diseases because it prevents the excessive energy storage and inflammation by dissipating energy through heat [21]. MHY2013 increased the fatty acid oxidation and browning-related gene expression and decreased the inflammatory gene expression in WAT of the obese mice. Thus, it is likely that the fatty acid oxidation-derived energy may be dissipated through heat generation as a result of MHY2013 treatment. Furthermore, the increase in fatty acid oxidation and thermogenic signaling in WAT may contribute to the notable decrease in circulating NEFA levels in the MHY2013-treated db/db mice. However, the mechanisms underlying MHY2013-mediated increase in WAT browning signaling are not directly examined in the current study. Considering other studies showing that all PPAR subtypes stimulate WAT browning with hormonedependent and -independent manners [21-24], we assume that the MHY2013-mediated increases in the expression of WAT browning markers and the formation of multi-locular lipid droplets in WAT are probably due to the MHY2013 agonism for all PPAR subtypes in multiple tissues and the PPAR agonism-dependent secretion of thermogenic activators, including FGF21 and adiponectin.

Although thermogenic signaling was increased in WAT, the body weight was unaltered in the mice treated with MHY2013. We assume that the WAT browning effect of MHY2013 may not be strong enough to reduce body weight or that other effects of all three PPAR subtype agonism by MHY2013 may offset the WAT browningmediated body weight regulation. For instance, FGF21 has been shown to decrease body weight and improve metabolic profile in obese mice $[25,26]$. On the other hand, adiponectin ameliorates metabolic profile although 
it increases body weight evidenced by clearly increased insulin sensitivity, healthier blood lipid profile, and less lipid accumulation in liver despite highly increased body weight in adiponectin-overexpressed ob/ob mice [27]. Thus, the net effect of MHY2013 may be the amelioration of metabolic profile without decreasing body weight.

In conclusion, the balanced activation of the three PPAR subtypes by MHY2013 improved obesity-induced insulin resistance, hepatic steatosis, and dyslipidemia without weight gain and severe toxicity. Although all the metabolic signaling pathways and target tissues are not examined in the current study, we assume that the elevation of beneficial hormones including FGF21 and adiponectin, WAT browning signaling, and fatty acid oxidation signaling in liver and skeletal muscle may synergistically contribute to the beneficial effects of MHY2013.

Our present study supports the concept of a pan PPAR therapeutic approach to conditions which comprise the metabolic syndrome. Further studies are needed to enable MHY2013 clinical use for targeting various metabolic diseases.

\section{MATERIALS AND METHODS}

\section{Animal experiments}

Male, 8-week-old C57BLKS/J-lean $(\mathrm{db} / \mathrm{m})$ and $\mathrm{C} 57 \mathrm{BLKS} / \mathrm{J}-\mathrm{db} / \mathrm{db}$ mice were purchased from Japan SLC and acclimated to the animal care facility for seven days before the experiments. Animals were housed in an airconditioned atmosphere under a $12 \mathrm{~h}$ light/dark cycle and were given free access to a standard rodent chow (Samtako) and water. The animal study was approved by the Institutional Animal Care Committee of Pusan National University (2, Busandaehak-ro 63beon-gil, Geumjeong-gu, Busan, Republic of Korea) and performed in accordance with the guidelines for animal experiments issued by Pusan National University. Vehicle and MHY2013 (5 mg/kg/day) were injected by oral gavage for three weeks. The mice were monitored daily for food intake and once in three days for body weight. After two weeks, a glucose tolerance test (GTT) was performed by intraperitoneal injection of $1.5 \mathrm{~g} / \mathrm{kg}$ glucose after overnight fasting. Blood was taken from the tail at indicated time points, and the glucose concentration was determined with a glucometer (Accu-Chek, Roche Diagnostics). The animals were euthanized after three weeks, and serum and tissues were collected.

\section{In Silico protein-ligand docking simulation}

The crystal structures of human $\operatorname{PPAR} \alpha, \operatorname{PPAR} \beta / \delta$, and PPAR $\gamma$ were obtained from the Protein Data Bank (PDB ID: PPAR $\alpha, 1 \mathrm{~K} 7 \mathrm{~L}$; PPAR $\beta / \delta, 1 \mathrm{GWX}$; and PPAR $\gamma$, $3 \mathrm{DZY}$ ) and used as the targets in docking calculations.
We used the AutoDock 4.2 program and the tool's manual because of its automated docking capabilities. To define the docking pockets of the three PPAR subtypes, we used a set of predefined active sites of human PPARs. Docking simulations were performed between the three PPAR subtypes and MHY2013. To prepare compounds for the docking simulation, we performed the following steps: [1] 2D structures were converted into 3D structures, [2] charges were calculated, and [3] hydrogen atoms were added using the ChemOffice program (http://www. cambridgesoft.com). In addition, the LigandScout 3.0 program was used to generate a pharmacophore model and to predict possible hydrogen-bonding residues between the three PPAR subtypes and MHY2013. AMBER ff99SB force-field parameter was applied for calculating ligand molecules. The docking protocol was validated by docking co-crystallized ligand structure. Energy evaluations were 2500000 and population size was 150 .

\section{Cell culture system and adipocytes differentiation}

YPEN-1, a rat prostate endothelial cell line, and HepG2, a human liver cancer cell line, were purchased from the American Type Culture Collection (Manassas, VA, USA). 3T3-L1, a murine pre-adipocyte cell line, was obtained from Dr. Hyeung-Rak Kim (Pukyoung National University, Pusan, Korea). Cells were maintained in Dulbecco's modified Eagle's medium containing 5\% (YPEN-1) or 10\% (HepG2 and 3T3-L1) fetal bovine serum (Gibco, Grand Island, NY, USA), $100 \mathrm{U} / \mathrm{mL}$ penicillin, and $100 \mu \mathrm{g} / \mathrm{mL}$ streptomycin (all from Hyclone, Inc., Logan, UT, USA) at $37^{\circ} \mathrm{C}$ in a $5 \% \mathrm{CO}_{2}$ atmosphere. For adipocytes differentiation, cells were cultured to confluence (day 0 ) and then exposed to the differentiation mixture (0.5 mm 3-isobutyl-1-methylxanthine, $1 \mu \mathrm{M}$ dexamethasone, and $10 \mu \mathrm{g} / \mathrm{mL}$ insulin). After $48 \mathrm{~h}$ of incubation, the cells were maintained in a medium containing $10 \mu \mathrm{g} / \mathrm{mL}$ insulin until harvest on day 7 .

\section{Primary adipocytes culture}

Subcutaneous fat pads from 8-week-old mice were excised, minced and digested in HEPES buffer with type 1 collagenase at $37{ }^{\circ} \mathrm{C}$ for $90 \mathrm{~min}$. The cells were diluted in 1\% FBS-DMEM buffer. Diluted cell suspension was filtered with $70 \mu \mathrm{m}$ nylon mesh to remove undigested tissues and then centrifuged at 500 $\mathrm{g}$ for $15 \mathrm{~min}$ to sediment clumps. The supernatant was removed and red blood cell (RBC) lysis buffer was added and gently suspended. After 5 min incubation at room temperature, equal volume of 10\% FBS-DMEM was added and then filtered with $40 \mu \mathrm{m}$ nylon mesh to remove endothelial cell clumps. Filtered cell suspension was centrifuged at $500 \mathrm{~g}$ for $5 \mathrm{~min}$ and all but $2-3 \mathrm{ml}$ of the supernatant was removed. The pellet was suspended 
with 10\% FBS-DMEM. The cell suspension was counted and seeded.

\section{Cell viability assay}

The cell viability assay was carried out using 3-(4,5-dimethylthiazol-2-yl)-2,5-diphenyltetrazolium bromide (MTT; DoGen, Korea). YPEN-1 cells $\left(1 \times 10^{4}\right)$ were plated in each well of a 96-well cell culture plate and allowed to attach overnight. After the cells were exposed to MHY2013 at concentrations ranging from 1 to $10 \mu \mathrm{M}$ for $24 \mathrm{~h}$, the MTT reagent was added to each well, and the plate was incubated for $1 \mathrm{~h}$. Absorbance in each well was determined at $560 \mathrm{~nm}$ using a microplate reader

\section{Luciferase assay}

For a peroxisome proliferator response element (PPRE)-driven luciferase assay, $5 \times 10^{4}$ YPEN-1 cells were seeded per well into a 48 -well cell culture plate. The PPRE-X3-TK-LUC plasmid $(0.5 \mu \mathrm{g})$ (a kind gift from Dr. Christoper K. Glass, University of California, San Diego, CA, USA) and $0.05 \mu \mathrm{g}$ of the PPAR $\alpha, \operatorname{PPAR} \beta / \delta$, and PPAR $\gamma$ expression vectors (kind gifts from Dr. Han Geuk Seo, Konkuk University, Seoul, South Korea) were transfected into the cells using $1 \mu \mathrm{L}$ of the Lipofectamine 2000 reagent (Invitrogen, Carlsbad, CA, USA) according to the manufacturer's instructions. For the FGF21luciferase assay, $2 \times 10^{4} \mathrm{HepG} 2$ cells were seeded per well into a 96-well cell culture plate. The FGF21-LUC plasmid $(0.1 \mu \mathrm{g})$ (a kind gift from Dr. Kook Hwan Kim, Yonsei University, Seoul, South Korea) was transfected into the cells using $0.1 \mu \mathrm{L}$ of the Lipofectamine 3000 reagent (Invitrogen) according to the manufacturer's instructions. After the incubation for $24 \mathrm{~h}$, the cells were treated with the vehicle[dimethyl sulfoxide (DMSO)], 10 $\mu \mathrm{M}$ MHY2013, WY14643 (a known PPAR $\alpha$ agonist), rosiglitazone (a known PPAR $\gamma$ agonist), and GW501516 (a known PPAR $\beta / \delta$ agonist) for $5 \mathrm{~h}$. Luciferase activity was measured using the One-Glo Luciferase Assay System (Promega, Madison, WI, USA) and a luminometer (Berthold Technologies GmbH \& Co., Bad Wildbad, Germany).

\section{Immunocytochemistry}

YEPN-1 cells $\left(2 \times 10^{5}\right)$ were seeded in a $35-\mathrm{mm}$ cover-glass-bottom dish and allowed to attach overnight. The cells were treated with $10 \mu \mathrm{M}$ MHY2013, WY14643, rosiglitazone, and GW501516 for $1.5 \mathrm{~h}$ and washed twice with phosphate-buffered saline (PBS). Then, the cells were fixed in 4\% paraformaldehyde for $30 \mathrm{~min}$ at room temperature and washed using PBS with $0.5 \%$ Triton X-100. After washing, the cells were blocked in ABS $/ 0.1 \%$ Triton X-100/3\% goat serum (ABS-TS) for $30 \mathrm{~min}$. Subsequently, the cells were immunostained with rabbit anti-PPAR $\alpha$, anti-PPAR $\beta / \delta$, and anti-PPAR $\gamma$ primary antibodies (all from Santa Cruz Biotechnology, Santa Cruz, CA, USA), diluted in ABS-TS $(1: 1,000)$, at $4{ }^{\circ} \mathrm{C}$ overnight. The cells were washed three times with ABS-TS, then incubated with a secondary anti-rabbit IgG antibody labeled with Alexa Fluor-488 (Invitrogen) for 30 min, and washed with ABS. Cell nuclei were visualized by immunostaining with Hoechst 33342 (Invitrogen), and immunostained images of the three PPAR subtypes were acquired by confocal laser scanning microscopy (TCS SP2, Leica, Wetzler, Germany).

\section{Protein extraction and immunoblot analysis}

For extraction of cytosol and nuclear fractions from cells and tissues, a cell pellet or tissue was suspended and homogenized in $10 \mathrm{mM}$ Tris, $\mathrm{pH} 8.0$, with $1.5 \mathrm{mM} \mathrm{MgCl}_{2}$, $1 \mathrm{mM}$ dithiothreitol, $0.1 \% \mathrm{NP}-40$, and protease inhibitors, incubated on ice for $15 \mathrm{~min}$, and then centrifuged at 12,000 rpm at $4{ }^{\circ} \mathrm{C}$ for $10 \mathrm{~min}$. The supernatants were used as the cytosolic fractions. The pellets were washed three times and re-suspended in $10 \mathrm{mM}$ Tris, $\mathrm{pH} 8.0$, with $50 \mathrm{mM}$ $\mathrm{KCl}, 100 \mathrm{mM} \mathrm{NaCl}$, and protease inhibitors, incubated on ice for $30 \mathrm{~min}$, and then centrifuged at $12,000 \mathrm{rpm}$ at $4{ }^{\circ} \mathrm{C}$ for $10 \mathrm{~min}$. The resultant supernatants were used as the nuclear fractions. The protein concentration was measured using the BCA assay (Thermo Scientific, Waltham, MA, USA). Samples were prepared in a gel buffer $(12.5 \mathrm{mM}$ Tris, 4\% sodium dodecyl sulfate, $20 \%$ glycerol, $10 \%$ 2-mercaptoethanol, and $0.2 \%$ bromophenol blue, $\mathrm{pH} 6.8$ ) and boiled for $5 \mathrm{~min}$. Western blot assays were performed as previously described, with minor modifications [28]. Primary antibodies raised against $\operatorname{PPAR} \alpha, \operatorname{PPAR} \beta / \delta$, PPAR $\gamma$, AMPK $\alpha 1 / 2$, phospho-AMPK $\alpha 1 / 2$ (Thr172), phospho-LKB1 (Ser431), transcription factor II B (TFIIB), and $\beta$-Actin (all from Santa Cruz Biotechnology) were used.

\section{Isolation of RNA and quantitative real-time polymerase chain reaction}

Tissue and cell RNA was purified using the TRIzol reagent (Invitrogen) according to the manufacturer's instruction. Total RNA $(2.0 \mu \mathrm{g})$ treated with RNase-free DNase was reverse-transcribed with a cDNA synthesis kit from GenDEPOT (Barker, TX, USA). Quantitative polymerase chain reaction (qPCR) was performed using the THUNDERBIRD SYBR qPCR mix (TOYOBO Co., Osaka, Japan) and a CFX Connect System (Bio-Rad Laboratories, Inc., Hercules, CA, USA). The primer sequences are shown in Supplementary Table 2.

\section{Serum biochemical analysis and cytokine measurements}

Serum glucose, cholesterol, triglycerides (TGs), non-esterified fatty acids (NEFAs), and creatinine were 
analyzed using kits from Bioassay Systems (Hayward, CA, USA). To measure the serum adiponectin level, the Mouse Adiponectin ELISA Kit (CircuLex Co.) was used. The serum fibroblast growth factor 21 (FGF21) level was evaluated using the Mouse/Rat FGF-21 Quantikine ELISA Kit (R\&D Systems). Serum alanine aminotransferase (ALT) and aspartate aminotransferase (AST) were measured using a kit from Stanbio (Boerne, TX, USA).

\section{Liver triglyceride measurements}

Liver samples were homogenized in ice-cold PBS. TGs were extracted with methanol/chloroform (1:2), dried, and suspended in 5\% bovine serum albumin. The TG level was evaluated using a kit from Bioassay Systems.

\section{Statistical analysis}

All data are expressed as the mean \pm standard error of the mean (SEM). Statistical significance of the differences between groups was determined by one-way analysis of variance, followed by a Dunnett's test. An associated probability ( $p$ value) of $<0.05$ was considered significant.

\section{ACKNOWLEDGMENTS}

We thank the Korean Aging Tissue Bank for providing research materials.

\section{CONFLICTS OF INTEREST}

The authors declare no conflicts of interest.

\section{GRANT SUPPORT}

This work was supported by the National Research Foundation of Korea (NRF) grant funded by the Korean government (MSIP) (NO. 2009-0083538).

\section{REFERENCES}

1. Daynes RA, Jones DC. Emerging roles of PPARs in inflammation and immunity. Nat Rev Immunol. 2002; 2:748-759.

2. Berger J, Moller DE. The mechanisms of action of PPARs. Annu Rev Med. 2002; 53:409-435.

3. Schoonjans K, Staels B, Auwerx J. The peroxisome proliferator activated receptors (PPARS) and their effects on lipid metabolism and adipocyte differentiation. Biochim Biophys Acta. 1996; 1302:93-109.

4. Schoonjans K, Staels B, Auwerx J. Role of the peroxisome proliferator-activated receptor (PPAR) in mediating the effects of fibrates and fatty acids on gene expression. J Lipid Res. 1996; 37:907-925.
5. Pourcet B, Fruchart JC, Staels B, Glineur C. Selective PPAR modulators, dual and pan PPAR agonists: multimodal drugs for the treatment of type 2 diabetes and atherosclerosis. Expert Opin Emerg Drugs. 2006; 11:379-401.

6. Lalloyer F, Staels B. Fibrates, glitazones, and peroxisome proliferator-activated receptors. Arterioscler Thromb Vasc Biol. 2010; 30:894-899.

7. Rubenstrunk A, Hanf R, Hum DW, Fruchart JC, Staels B. Safety issues and prospects for future generations of PPAR modulators. Biochim Biophys Acta. 2007; 1771:1065-1081.

8. Balakumar P, Rose M, Ganti SS, Krishan P, Singh M. PPAR dual agonists: are they opening Pandora's Box? Pharmacol Res. 2007; 56:91-98.

9. Lee WH, Kim SG. AMPK-Dependent Metabolic Regulation by PPAR Agonists. PPAR Res. 2010; 2010.

10. Xu J, Lloyd DJ, Hale C, Stanislaus S, Chen M, Sivits G, Vonderfecht S, Hecht R, Li YS, Lindberg RA, Chen JL, Jung DY, Zhang Z, et al. Fibroblast growth factor 21 reverses hepatic steatosis, increases energy expenditure, and improves insulin sensitivity in diet-induced obese mice. Diabetes. 2009; 58:250-259.

11. Etgen GJ, Oldham BA, Johnson WT, Broderick CL, Montrose CR, Brozinick JT, Misener EA, Bean JS, Bensch WR, Brooks DA, Shuker AJ, Rito CJ, McCarthy JR, et al. A tailored therapy for the metabolic syndrome: the dual peroxisome proliferator-activated receptoralpha/gamma agonist LY465608 ameliorates insulin resistance and diabetic hyperglycemia while improving cardiovascular risk factors in preclinical models. Diabetes. 2002; 51:1083-1087.

12. Teramoto T, Shirai K, Daida H, Yamada N. Effects of bezafibrate on lipid and glucose metabolism in dyslipidemic patients with diabetes: the J-BENEFIT study. Cardiovasc Diabetol. 2012; 11:29.

13. Monk JP, Todd PA. Bezafibrate. A review of its pharmacodynamic and pharmacokinetic properties, and therapeutic use in hyperlipidaemia. Drugs. 1987; 33:539-576.

14. Guo Y, Jolly RA, Halstead BW, Baker TK, Stutz JP, Huffman M, Calley JN, West A, Gao H, Searfoss GH, Li $\mathrm{S}$, Irizarry AR, Qian HR, et al. Underlying mechanisms of pharmacology and toxicity of a novel PPAR agonist revealed using rodent and canine hepatocytes. Toxicol Sci. 2007; 96:294-309.

15. Park MH, Park JY, Lee HJ, Kim DH, Park D, Jeong HO, Park CH, Chun P, Moon HR, Chung HY. Potent antidiabetic effects of MHY908, a newly synthesized PPAR alpha/gamma dual agonist in $\mathrm{db} / \mathrm{db}$ mice. PLoS One. 2013; 8:e78815.

16. Park MH, Kim DH, Kim MJ, Lee EK, An HJ, Jeong JW, Kim HR, Kim SJ, Yu BP, Moon HR, Chung HY. Effects of MHY908, a New Synthetic PPARalpha/gamma Dual Agonist, on Inflammatory Responses and Insulin Resistance 
in Aged Rats. J Gerontol A Biol Sci Med Sci. 2016; 71:300-309.

17. Muise ES, Azzolina B, Kuo DW, El-Sherbeini M, Tan Y, Yuan X, Mu J, Thompson JR, Berger JP, Wong KK. Adipose fibroblast growth factor 21 is up-regulated by peroxisome proliferator-activated receptor gamma and altered metabolic states. Mol Pharmacol. 2008; 74:403-412.

18. Yang C, Lu W, Lin T, You P, Ye M, Huang Y, Jiang X, Wang C, Wang F, Lee MH, Yeung SC, Johnson RL, Wei C, et al. Activation of Liver FGF21 in hepatocarcinogenesis and during hepatic stress. BMC Gastroenterol. 2013; 13:67.

19. Maeda N, Takahashi M, Funahashi T, Kihara S, Nishizawa H, Kishida K, Nagaretani H, Matsuda M, Komuro R, Ouchi N, Kuriyama H, Hotta K, Nakamura T, et al. PPARgamma ligands increase expression and plasma concentrations of adiponectin, an adipose-derived protein. Diabetes. 2001; 50:2094-2099.

20. Srivastava RA, Pinkosky SL, Filippov S, Hanselman JC, Cramer CT, Newton RS. AMP-activated protein kinase: an emerging drug target to regulate imbalances in lipid and carbohydrate metabolism to treat cardio-metabolic diseases. J Lipid Res. 2012; 53:2490-2514.

21. Ohno H, Shinoda K, Spiegelman BM, Kajimura S. PPARgamma agonists induce a white-to-brown fat conversion through stabilization of PRDM16 protein. Cell Metab. 2012; 15:395-404.

22. Barbera MJ, Schluter A, Pedraza N, Iglesias R, Villarroya F, Giralt M. Peroxisome proliferator-activated receptor alpha activates transcription of the brown fat uncoupling protein-1 gene. A link between regulation of the thermogenic and lipid oxidation pathways in the brown fat cell. J Biol Chem. 2001; 276:1486-1493.
23. Rachid TL, Penna-de-Carvalho A, Bringhenti I, Aguila MB, Mandarim-de-Lacerda CA, Souza-Mello V. Fenofibrate (PPARalpha agonist) induces beige cell formation in subcutaneous white adipose tissue from dietinduced male obese mice. Mol Cell Endocrinol. 2015; 402:86-94.

24. Ngala RA, Stocker CJ, Roy AG, Hislop D, Wargent E, Bell R, Hassall DG, Harling JD, Billin AN, Willson TM, Arch JR, Cawthorne MA. A new, highly selective murine peroxisome proliferator-activated receptor delta agonist increases responsiveness to thermogenic stimuli and glucose uptake in skeletal muscle in obese mice. Diabetes Obes Metab. 2011; 13:455-464.

25. Owen BM, Ding X, Morgan DA, Coate KC, Bookout AL, Rahmouni K, Kliewer SA, Mangelsdorf DJ. FGF21 acts centrally to induce sympathetic nerve activity, energy expenditure, and weight loss. Cell Metab. 2014; 20:670-677.

26. Straub L, Wolfrum C. FGF21, energy expenditure and weight loss - How much brown fat do you need? Mol Metab. 2015; 4:605-609.

27. Kim JY, van de Wall E, Laplante M, Azzara A, Trujillo ME, Hofmann SM, Schraw T, Durand JL, Li H, Li G, Jelicks LA, Mehler MF, Hui DY, et al. Obesity-associated improvements in metabolic profile through expansion of adipose tissue. J Clin Invest. 2007; 117:2621-2637.

28. Jung YR, Kim DH, Kim SR, An HJ, Lee EK, Tanaka T, Kim ND, Yokozawa T, Park JN, Chung HY. Anti-wrinkle effect of magnesium lithospermate B from Salvia miltiorrhiza BUNGE: inhibition of MMPs via NF-kB signaling. PLoS One. 2014; 9:e102689. 